\title{
SCALING PHENOMENA IN FATIGUE AND FRACTURE
}

\author{
G.I. Barenblatt \\ Department of Mathematics \\ University of California and Lawrence Berkeley National Laboratory \\ Berkeley, CA 94720-3840, USA
}

\begin{abstract}
The general classification of scaling laws will be presented and the basic concepts of modern similarity analysis - intermediate asymptotics, complete and incomplete similarity — will be introduced and discussed. The examples of scaling laws corresponding to complete similarity will be given. The Paris scaling law in fatigue will be discussed as an instructive example of incomplete similarity. It will be emphasized that in the Paris law the powers are not the material constants. Therefore, the evaluation of the life-time of structures using the data obtained from standard fatigue tests requires some precautions.
\end{abstract}

\section{Introduction}

Signore il Presidente, Ladies and Gentlemen,

\section{Scaling laws}

$$
y=C x_{1}^{\alpha_{1}} \ldots x_{n}^{\alpha_{n}}
$$

which often appear in modeling phenomena in nature, engineering and society, seem to be trivial. Very often they are considered only as a first, simplest attempt to represent a set of data. Indeed, laws are trivial, but non-trivial is why they are trivial! The reason of their importance is that the scaling laws always reveal a deep feature of processes under consideration: self-similarity. For processes developing in time self-similarity means that the phenomenon is reproducing itself in scales, which vary in time:

$$
\mathbf{U}(\mathbf{r}, t)=\mathbf{U}_{0}(t) f\left(\frac{\mathbf{r}}{\ell(t)}\right) .
$$

Establishing scaling laws and self-similarity was always considered as an important, sometimes crucially important step in construction of engineering and physical theories. In the pre-computer era they were considered as special, "exact" solutions illuminating complicated models, elegant, sometimes useful, but nevertheless restricted in their value, elements of theories. Later, when computers entered into play, the role of such solutions did not diminish, just the contrary. However, the general attitude to them changed: they started to attract attention mainly as "intermediate asymptotics" — an important element of physical or engineering theories describing the behavior of systems when the 
influence of accidental details of the initial and/or boundary conditions already disappears, but the system is still far from the ultimate state of equilibrium.

Establishing the scaling laws and self-similarities was of special importance in studies of turbulence and structural strength. Remarkably both these branches of natural and engineering science were started by two great Italians: Leonardo da Vinci, born in a suburb of Florence, and Galileo Galilei, born in Pisa: both these places are not far from Torino where we are assembled these days.

Turbulence is the fluid flow with randomly accumulating and dissipating vortices.

Structural strength is inseparable from fatigue. Fatigue in a broader sense is the deformation and flow of solids with accumulating defects, terminating by fracture.

Generally in scientific circles turbulence is considered as a major challenge of applied mathematics and classical physics. Among those who made seminal contributions to studies of turbulence were giants - A.N. Kolmogorov and W. Heisenberg, as well as great applied mathematicians of the XX century - L. Prandtl, Th. von Kármán and G.I. Taylor.

At the same time in the circles of physicists and applied mathematicians the problem of structural strength, and in particular the problem of fatigue, definitely remains overshadowed by turbulence. Meanwhile, fatigue and fracture present not less thrilling fundamental problems than turbulence, not to speak about practical importance. In fact, the phenomenon of fatigue is in principle even more complicated! Indeed, stop the turbulent flow of air and/or water, and the fluid becomes indistinguishable from that at the beginning of the motion. This is not the case for fatigue. And there is even something worse. In turbulence we have good experimental reasons to believe that the fluid, like air and water remains Newtonian even in the most complicated flows up to the scales of smallest vortices. At the same time even for quasi-brittle solids the very possibility of using any constitutive equations for the materials near the defects is doubtful!

Parallels between turbulence and fatigue are instructive - also in another aspect. Yes, turbulence is generally considered as a great challenge, and during more than a century an army of engineers and scientists led by geniuses attacked it. But let's ask ourselves with full sincerity: what was achieved during this time as far as the creation of pure self-contained theory of turbulence based on first principles is concerned? The answer is very disappointing - practically nothing! And it is clear now that such a theory will not be constructed in real time such was in particular the opinion of A.N. Kolmogorov. He claimed that the practical way is to construct models based on special hypotheses relying on the results of experimental studies. Clearly such models could be valid for special classes of flows only. It can be expected that the same path is to be followed in structural strength and fatigue studies.

Here I want to emphasize that practically all significant results in turbulence studies were obtained using similarity considerations and scaling laws. The value of these tools and their technique should be properly understood, and they should be properly used in everyday practice by engineers and researchers 
working in fatigue and fracture.

This is the main topic for my lecture. It is well known that the subject of scaling in structural strength attracted the attention of engineers and researchers starting from Galileo. There exist multiple treatises, monographs and papers where the scaling problems in structural strength are treated. I want to mention especially recent monographs and papers by Professor Z. Bažant and his school [1-6], the very recent summarizing paper [6] should be especially recommended - and our President Professor A. Carpinteri and his school [7-13]. We all learned a lot from their remarkable works. However, I do not attempt to review and discuss them here in this lecture - it would be impossible. We have a special section to discuss the problems of scaling in structural engineering, where their contributions will find a descent place.

In my lecture I will speak about the results related to a different approach, developed independently starting from the late fifties-early sixties in my close collaboration with my unforgettable colleague and friend Ya. B. Zeldovich [14,15]. My students and close colleagues G.I. Sivashinsky and L.R. Botvina participated in various parts of these works.

\section{Scaling laws obtained by dimensional analysis}

I will present here the state of art of these matters with some instructive examples of scaling laws and scaling phenomena. Naturally all except one of the examples will be related to fracture and fatigue, the examples from other fields in particular turbulence can be found in my books $[16,17]$. However, I want to make a short intermezzo: to demonstrate one of the milestones not only of fluid mechanics, but of engineering science as a whole achieved by similarity methods. I mean the famous scaling law obtained first by Sir Geoffrey Taylor [18] for the radius $r_{f}$ of the shock wave formed after an atomic explosion (Figure 1):

$$
r_{f}=\left(\frac{E t^{2}}{\rho_{0}}\right)^{1 / 5} .
$$

Here $E$ is the energy of the explosion, $t$ - the time after the explosion, and $\rho_{0}$ - the air density before the explosion. Scaling law (3) was obtained by G.I. using dimensional analysis (I will demonstrate this technique on examples from fracture and fatigue later, the explanation of it with details and examples can be found in $[16,17]$.) I emphasize specially: of crucial importance was not the formal application of rather simple rules of dimensional analysis, but the preliminary idealization of the problem which was invented by G.I. Taylor: He made two basic assumptions - the explosion is instantaneous and concentrated at a point. Furthermore, the initial atmospheric pressure $p_{0}$ is negligible in comparison with the pressure behind the shock wave. This basic idealization allowed G.I. Taylor to establish the self-similarity of the phenomenon and to assume that the radius of the shock wave $r_{f}$ can depend on $E$, $t$, and $\rho_{0}$ only. After this assumption 


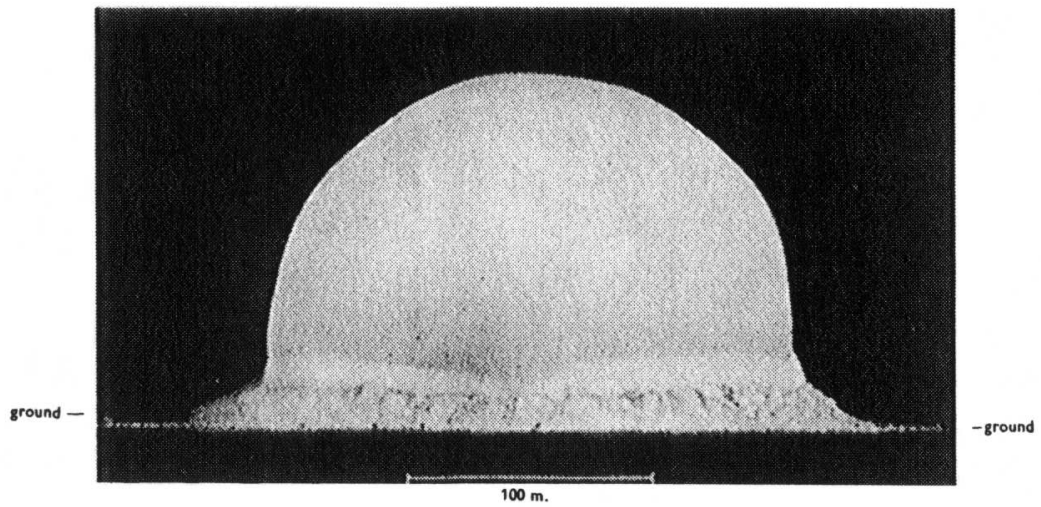

Figure 1: Photograph of the fireball of the atomic explosion.

scaling law (3) followed from dimensional analysis immediately. Scaling law (3) obtained excellent experimental confirmation ([19], Figure 2).

In fracture mechanics also there are well-known scaling laws which can be obtained after a plausible idealization in G.I. Taylor style using dimensional analysis. I want to mention here some of them. The first example is the symmetric wedging of a brittle or quasi-brittle thick plate (plain strain) by a thin wedge $([20])$, see Figure 3a.

We introduce a natural idealization. First we assume that the spearheads of the wedge are concentrated on opposite lines, so that the action of the wedge is represented by two equal and oppositely directed concentrated tractions $P$, per unit length of the plate thickness (Figure $3 \mathrm{~b}$ ). Further, we assume that the plate is infinite. Thus, the length of the crack $\ell$ can depend only on $P$, the Poisson ratio $\nu$, and a characteristic of fracture toughness, the cohesion modulus $K$. I emphasize here the known difference between the cohesion modulus $K$, introduced by the author in 1959 [20], and determined under conditions of stable crack propagation, and another fracture toughness characteristic $K_{I c}$, introduced practically simultaneously by G.R. Irwin in 1960 [21] and determined by the beginning of the unstable crack propagation. In the case under consideration the crack is stable, therefore a relation for the crack length $\ell$ is valid

$$
\ell=f(P, K, \nu) .
$$

The dimensions of the quantities entering relation (4) are obviously as follows:

$$
[\ell]=L, \quad[P]=F L^{-1}, \quad[K]=F L^{-3 / 2}, \quad[\nu]=1
$$

where $L$ and $F$ are the dimensions of the length and force. From the arguments only $P$ and $K$ have independent dimensions, and only one quantity of the dimension of length can be formed of them: $P^{2} / K^{2}$. Therefore the dimensionless 


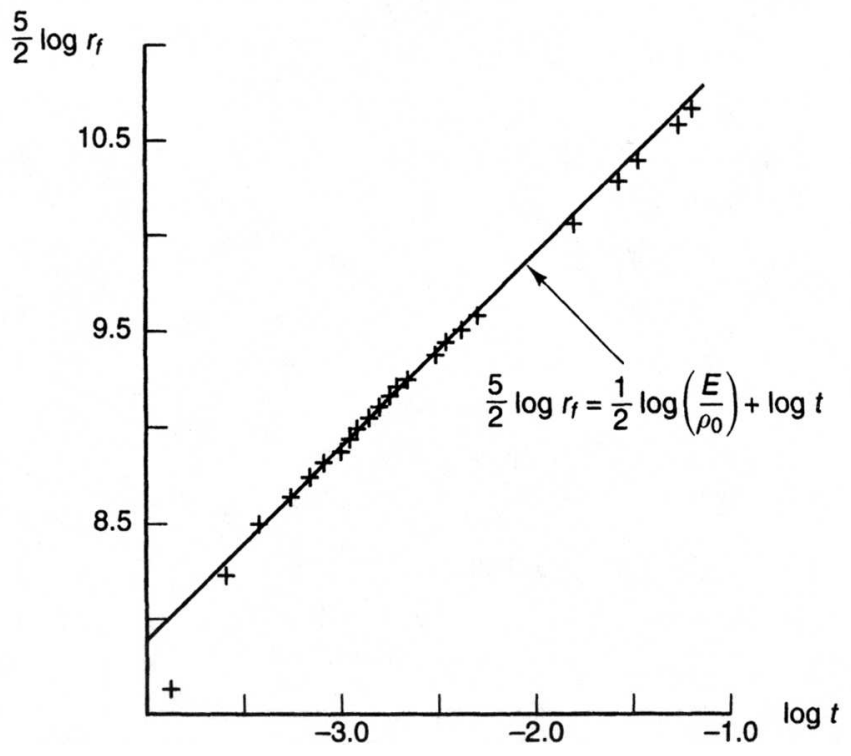

Figure 2: The experimental results confirms scaling law (3) (Taylor [19])

quantity $\ell /\left(P^{2} / K^{2}\right)$ can depend on the Poisson ratio $\nu$ only, and we obtain

$$
\ell=\operatorname{Const}(\nu) \frac{P^{2}}{K^{2}} .
$$

An analytic calculation [20] shows that Const is equal to one. Scaling law (5) also obtained a reliable experimental confirmation, and relation (5) served for experimental evaluation of the cohesion modulus (see the comprehensive book by V.V. Panasyuk [22].

The cohesion crack model was introduced by the author in 1959 [20]. Before that the analogous problem was considered by the author [23] for the case when the propagation of the crack is resisted by uniform compression $q$, like in rock massives (also an instructive illustrative example is achieved by a ruler inserted between the pages of a horizontally lying book). In this case the scaling law is different:

$$
\ell=\frac{2}{\pi} \frac{P}{q}
$$

so that the length of the crack is proportional to the first, not the second, degree of the load.

A remarkable scaling law was obtained using the dimensional analysis by F. Roesler [24], and J.J. Benbow [25] for the cone crack formed under small diameter punch in a block of fused silica (Figure 4). Roesler and Benbow also replaced the problem by an idealized one: point indentor punched into an infinite 

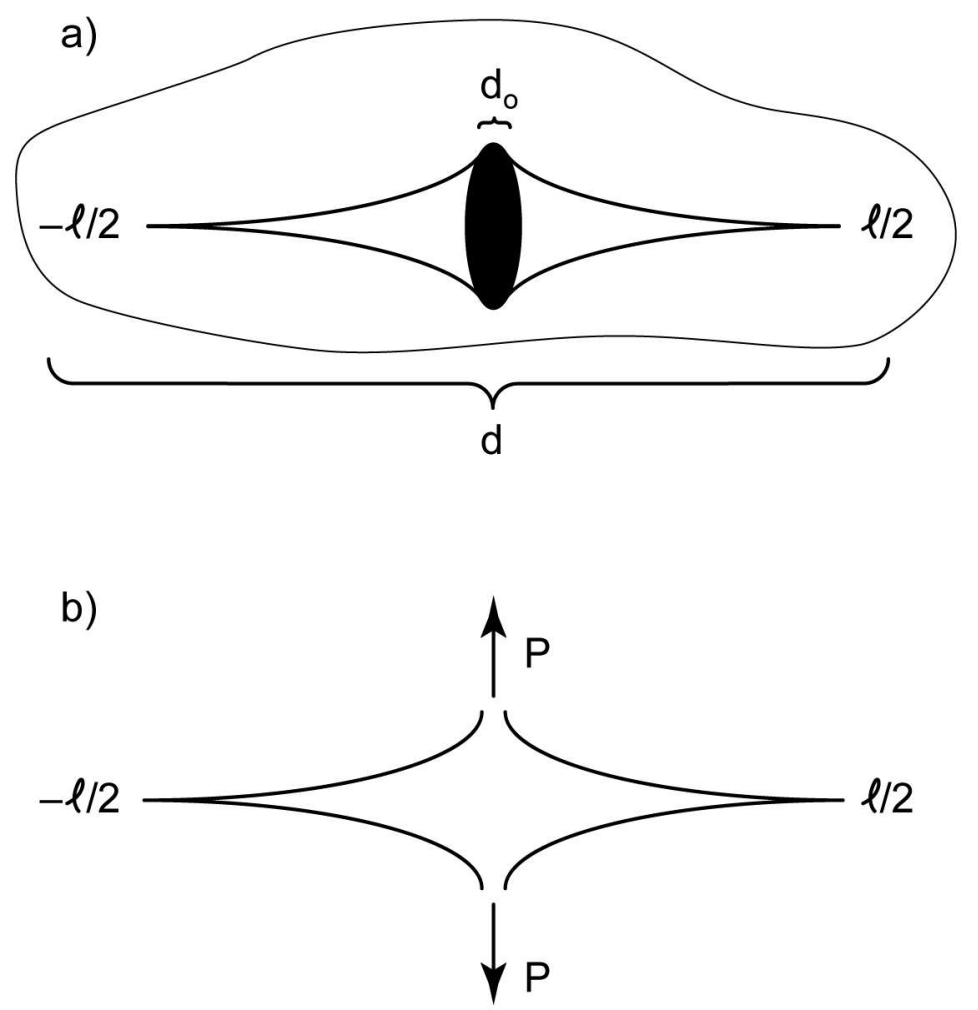

Figure 3: The wedging of a thick plate. (a) Original problem. (b) Idealized problem. 


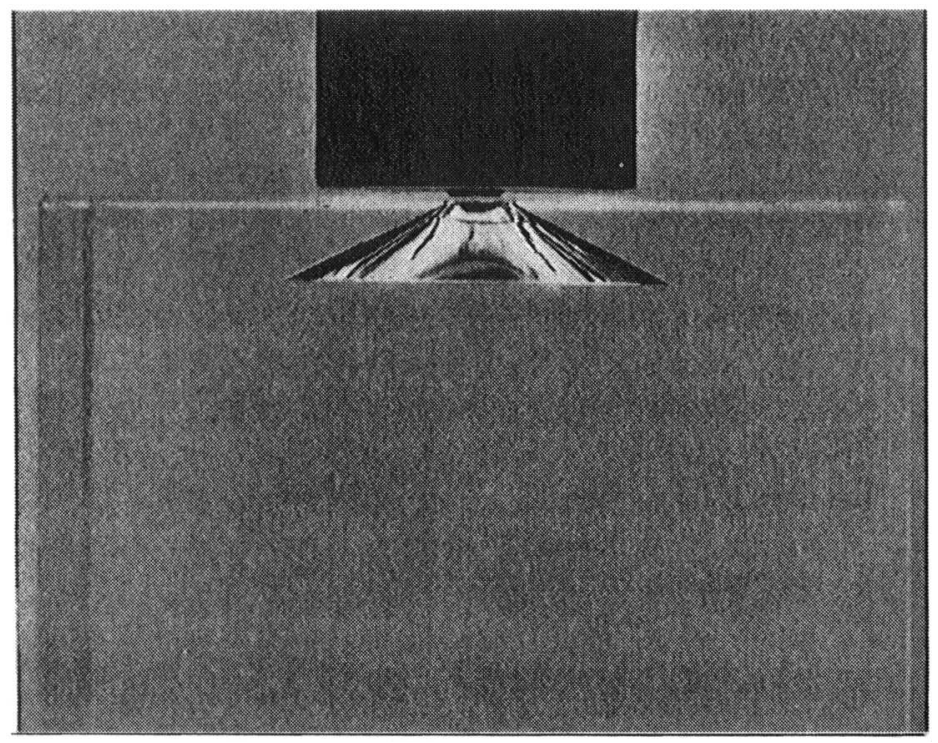

Figure 4: Conical crack in a fused silica block (Benbow [25]).

block. The scaling law, also obtained by dimensional analysis, for the diameter of the base of the cone crack $D$ under the load $P$ has the form:

$$
D=\operatorname{Const}(\nu)\left(\frac{P}{K}\right)^{2 / 3} \text {. }
$$

This scaling law also obtained remarkable experimental confirmation (Figure $5)$.

When considering the examples presented above, a natural question can arise: In fact, for instance, for the problem of wedging of a thick plate, there appear two additional length scales: the width of the spearhead of the wedge $d_{0}$ which we equal to zero, and the size of the block $d$, which was assumed to be infinite. However, in fact, both these length scales are finite, and if we take them into account in relation (4), the dimensional analysis will lead to a different, and completely non-constructive result:

$$
\begin{aligned}
D & =\left(\frac{P}{K}\right)^{2} \Phi\left(\Pi_{1}, \Pi_{2}, \nu\right) \\
\Pi_{1} & =\frac{d_{0}}{\left(\frac{P^{2}}{K^{2}}\right)}, \quad \Pi_{2}=\frac{d}{\left(\frac{P^{2}}{K^{2}}\right) .}
\end{aligned}
$$

In fact, scaling law (6) is an "intermediate-asymptotic law" valid when

$$
d_{0} \ll D \ll d .
$$




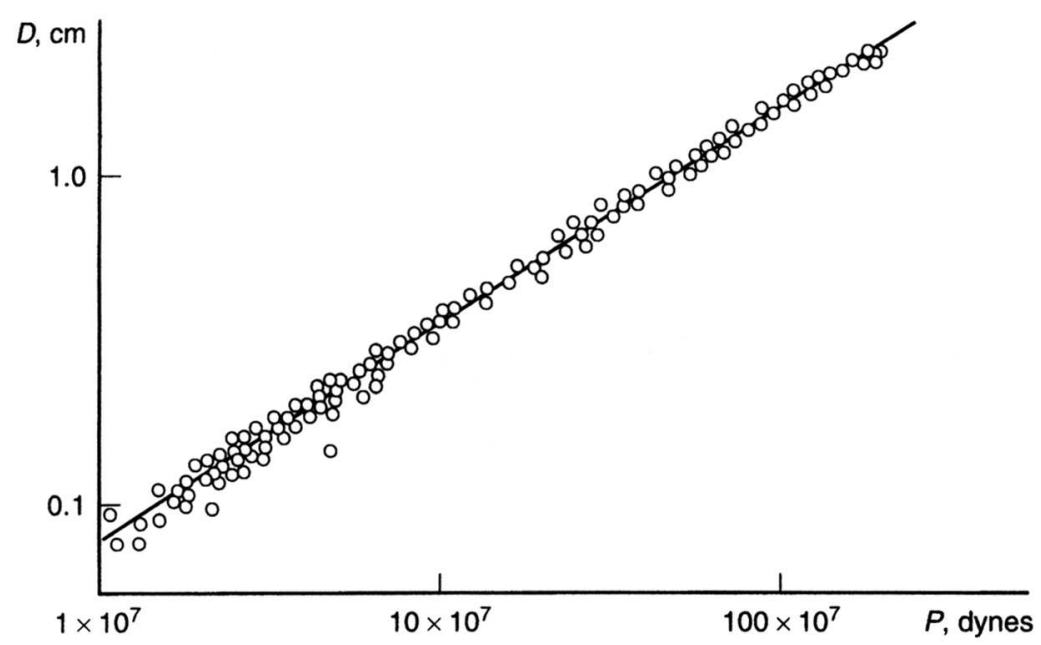

Figure 5: The experimental data confirm scaling law (7) (Benbow [25]).

The decisive advantage of the problem of wedging of a plate is that it can be proved rigorously that there exists a finite, non-zero limit of the function $\Phi\left(\Pi_{1}, \Pi_{2}, \nu\right)$, at $\Pi_{1} \rightarrow 0$ and $\Pi_{2} \rightarrow \infty$. Therefore at sufficiently small $\Pi_{1}$, and sufficiently large $\Pi_{2}$ the intermediate asymptotic scaling law (5) works. Apparently, excellent experimental confirmation of scaling laws (3) and (7) shows that the same situation happens in G.I. Taylor and Roesler-Benbow problems, although up to the present time nobody has proved it rigorously.

But what is the practical meaning of this fact? It means that if we take a different larger or smaller wedge, and a larger or smaller block from the same material, so that $d_{0}^{\prime}=\lambda d_{0}, d^{\prime}=\mu d$, where $\lambda$ and $\mu$ are certain positive numbers of the order of unity, nothing will change at this intermediate stage! Similarly, if we take a smaller or larger punch and a smaller or larger block in the RoeslerBenbow problem the propagation of the crack at the intermediate stage will not change. Also, in G.I. Taylor problem of very intense explosion the moderate variation of the charge size and of the atmospheric pressure will change noting in the propagation of the shock wave.

These are simple examples of the invariance of the intermediate asymptotics with respect to the renormalization group, a concept of extreme importance in the whole of this subject. The details concerning the renormalization group and its connection with intermediate asymptotics can be found in the books of Goldenfeld [26] and the author $[16,17]$. 


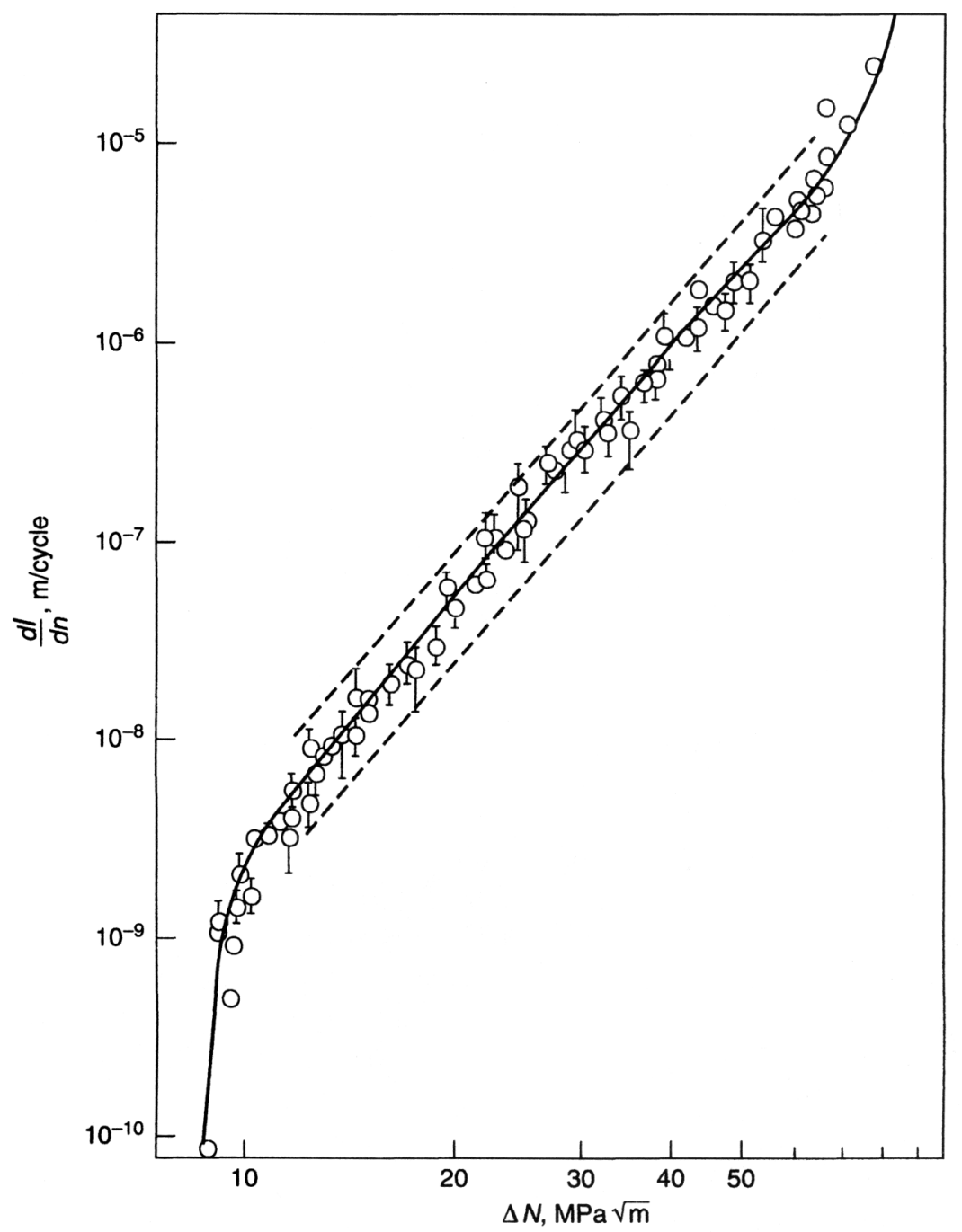

Figure 6: Experimental data for fatigue crack growth in the alluminum alloy confirm Paris law (17) in the major part of the crack velocity range (Botvina $[38])$. 


\section{Classification of scaling laws: complete and incomplete similarity}

A natural question arises. There exist many scaling laws in engineering science and physics. Whether the idyllic situation demonstrated above on three examples is always the case, and all scaling laws can be obtained using dimensional analysis after a simple natural idealization-like point explosion or pressing along a line in an infinite space. The answer is no, and there should be no illusions: as a rule the scaling laws and self-similarities cannot be obtained by dimensional analysis alone, and such "natural" idealization does not exist. In particular, this is true for a basic scaling law in fatigue: the Paris law; we will see it later.

To clarify this point, let's consider the scaling laws from a more general viewpoint. Namely, consider a certain physical relation, i.e., a relation, valid for all observers having different sizes of units of measurement of the same physical nature:

$$
a=f \overbrace{\left(a_{1}, \ldots, a_{k}, b_{1}, b_{2} \ldots\right)}^{n \text { arguments }} .
$$

Here $a$ is the quantity, possibly a vector, to be determined, $a_{1}, \ldots, a_{k}$ - the arguments, having independent dimensions, like say length, time, density whereas the dimensions of $a, b_{1}, b_{2}$ - are dependent, and can be expressed via the dimensions of $a_{1}, \ldots, a_{k}$ :

$$
[a]=\left[a_{1}\right]^{p} \ldots\left[a_{k}\right]^{r} ; \quad\left[b_{1}\right]=\left[a_{1}\right]^{p_{1}} \ldots\left[a_{k}\right]^{r_{1}} \ldots .
$$

Dimensional analysis allows one to reduce relation (10) to a dimensionless form:

$$
\begin{aligned}
\Pi & =\Phi \overbrace{\left(\Pi_{2}, \Pi_{2} \ldots\right)}^{n-k \text { arguments }} \\
\Pi & =\frac{a}{a_{1}^{p} \ldots a_{k}^{r}}, \quad \Pi_{1}=\frac{b_{1}}{a_{1}^{p_{1}} \ldots a_{k}^{r_{1}}}, \ldots
\end{aligned}
$$

The advantage of relation (12) in comparison with the original relation (10) is that the number of arguments of function $\Phi$ in (12) is less than the number

of arguments of function $f$ in the original equation (10). Moreover, according to (12) the basic function $f$ entering a physical relation possesses an important property of generalized homogeneity, i.e., it can be represented via a function $\Phi$ of a lesser number of arguments:

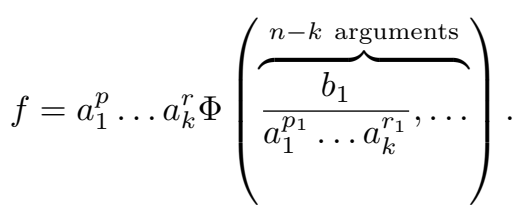

When we idealized the problems considered in the previous section, we did not intend to consider the limiting cases $\Pi_{1}=0, \Pi_{2}=\infty$. What we really did 
— we obtained the asymptotics (intermediate asymptotics), i.e., the asymptotic relations which appear when $\Pi_{1}, \Pi_{2}, \ldots$ are small or large, but not equal to their limiting values.

If there exists a finite non-zero limit $C$ of the function $\Phi$ at $\Pi_{1}, \Pi_{2} \ldots \rightarrow 0, \infty$, then at sufficiently small (large) $\Pi_{1}, \Pi_{2} \ldots$ the function $\Phi$ can be replaced by its limiting value $C$, and the scaling law is valid:

$$
a=f=C a_{1}^{p} \ldots a_{k}^{r}
$$

so that the arguments $b_{1}, b_{2} \ldots$ disappear from the resulting relation, and all powers $p, \ldots, r$ can be obtained by a simple procedure of dimensional analysis, as it was demonstrated in the problems considered in the previous section.

Colleagues, we say often, teaching our students, and reducing the relations to a dimensionless form, that if a dimensionless argument is small or large, it can be neglected. Generally speaking this statement is erroneous; it is correct only when the finite non-zero limit of the function $\Phi$ in (12) does exist, which is a priori unknown. And what will happen if such a finite non-zero limit of the function $\Phi$ does not exist, which is in fact the general case? In this case we cannot say anything in general terms, if, of course, we do not know the solution, in which case the similarity approach is superfluous.

However, the following remarkable possibility should not be missed. Let the finite non-zero limit of the function $\Phi$ not exist, but at small (large) $\Pi_{1}, \Pi_{2}, \ldots$ let the function $\Phi$ possess in its turn the property of generalized homogeneity in its dimensionless arguments. Here we consider the simplest case (for the general discussion the reader can be addressed to the books $[16,17])$ which we will need further:

$$
\Phi=\Pi_{1}^{\alpha} \Phi_{1}+\ldots
$$

where this time the function $\Phi_{1}$ has a finite non-zero limit at $\Pi_{1}, \Pi_{2}, \ldots \rightarrow 0, \infty$. Substitute (14) to (12), and we obtain that in this case again at large (small) values of $\Pi_{1}, \ldots$ a scaling law of type (14) is valid:

$$
a=f=C a_{1}^{p-\alpha p_{1}} \ldots a_{k}^{r-\alpha r_{1}} b_{1}^{\alpha} .
$$

There is however a substantial difference between scaling laws (14) and (16):

(a) The exponents $p-\alpha p_{1} \ldots r-\alpha r_{1}$ cannot be obtained by using dimensional analysis alone, because the power $\alpha$ in (15) is unknown. It should be obtained using some additional information, including sometimes experimental or computational data;

(b) The argument $b_{1}$ does not disappear from scaling law (16), it continues to influence the phenomenon, although it enters only in multiplicative combination with other parameters;

(c) The generalized homogeneity of function $\Phi$, contrary to the case of function $f$ in the original relation (10), does not follow from a fundamental physical principle of equality of all observers having different magnitude of the units of measurement of the same physical nature. Just the contrary: this is a property only of the special phenomenon under consideration. 
In the case when the scaling law of type (14) is valid we speak about the complete similarity of the phenomenon in the parameters $\Pi_{1}, \Pi_{2}, \ldots$ ( $\mathrm{cf}$ Reynolds number similarity in turbulence, the generally accepted term for the situation when the influence of the Reynolds number disappears).

In the second case when the scaling law of type (16) is valid we speak about incomplete similarity. Clearly, incomplete similarity is a much more general case than complete similarity, although it is still a very special case of asymptotic laws at small (large) values of parameters $\Pi_{1}, \Pi_{2} \ldots$ Discovery of incomplete similarity in gas dynamics, turbulence and fatigue marked important steps in the development of these disciplines. Mandelbrot fractals [27-29] also present a remarkable example of incomplete similarity. Incomplete similarity is also related to the asymptotic invariance of the mathematical model to a renormalization group, but in this case the renormalization group is more complicated. The details can be found in the books $[16,17]$.

\section{Paris law - an example of incomplete simi- larity}

An instructive and important example of incomplete similarity is represented by the Paris scaling law in fatigue.

The standard fatigue experiment is performed as follows. A specimen (notched or slotted bar or plate) is loaded by a combination of static tensile and a pulsating tensile load of constant frequency and amplitude. At the tip of the notch a fatigue crack is formed, and its propagation, i.e., its length as a function of the number of cycles $n$ is recorded. The Paris law is specified for multicycle fatigue tests, when the number of cycles before failure is of the order of many millions.

Processing the experimental data of such tests [30] (see also the preceding paper [31]) revealed the following scaling law

$$
\frac{d \ell}{d n}=A(\Delta N)^{m}
$$

which is being established after a certain initial stage. Here $d \ell / d n$ is the crack speed averaged over the cycle; $\Delta N=N_{\max }-N_{\min }$ is the stress-intensity-factor amplitude, $A$ and $m$ are empirically obtained constants. Under the conditions of the present experiment $N=C \sigma \sqrt{\ell}$, where $\sigma$ is the pulsating bulk stress, and the constant $C$ is a form-factor which can be evaluated using the technique of the theory of elasticity. The Paris law (17) has found subsequently multiple confirmations for different materials (see example in Figure 6) and now is considered as one of the fundamental laws of structural strength engineering science.

And here an important and very practical question arises: whether $A$ and $m$ are material constants, or may they be different for different specimens? The constant $m$ was found to vary in a wide range from slightly more than two to ten and even more. In engineering practice, scaling law (17) is used for an 
important prediction of the life-time of the structure, i.e., the number of cycles before failure $n_{F}$. Relation (17) can be rewritten in the form:

$$
\frac{d \ell}{d n}=A C^{m}\left(\sigma_{\max }-\sigma_{\min }\right)^{m} \ell^{\frac{m}{2}} .
$$

By integration we obtain:

$$
\frac{1}{\ell_{0}^{\frac{m}{2}}-1}-\frac{1}{\ell^{\frac{m}{2}}-1}=G(m-2) n
$$

where $G=A C^{m}\left(\sigma_{\max }-\sigma_{\min }\right)^{m} / 2$, and $\ell_{0}$ - the initial crack length. Remember that in multicycle fatigue the number of cycles before the failure is very high, so that it is possible to neglect in the evaluation of the life-time the number of cycles corresponding to the preliminary stage when the Paris law (17) still does not hold.

So, because the intermediate self-similar stage where the Paris law is valid holds during the basic part of the fatigue fracture process, the estimate for the life-time, i.e., for the number of cycles before the failure $n_{F}$ can be obtained from (19) assuming $\ell \gg \ell_{0}$ and neglecting the second term in the left-hand side of (19). We obtain

$$
n_{F}=\frac{1}{(m-2) G \ell_{0}^{\frac{m-2}{2}}} .
$$

Clearly the life-time $n_{F}$ sharply depends upon the parameter $m$, the exponent entering the Paris law, and also upon the pre-power coefficient $A$, entering expression (17) of this law. Therefore, it is worthwhile to analyze the Paris scaling law (17) on the basis of the procedure outlined above to understand whether these coefficients are indeed the material constants.

We assume that the shape of the loading cycle is fixed. Then the dependent quantity, the mean crack velocity averaged over the cycle $d \ell / d n$ can depend in principle upon the following arguments: the stress-intensity-factor amplitude $\Delta N=N_{\max }-N_{\min }$, stress-intensity-factor asymmetry $R=N_{\max } / N_{\min }$ (as a reminder, $N_{\max }$ and $N_{\min }$ are the maximum and minimum values of the stress intensity factor over the cycle), and, what is specially important, the characteristic length scale of the specimen $h$, e.g., its diameter, or thickness. The number of cycles $n$ does not enter the list of the governing parameters because it is the intermediate self-similar stage under consideration. Also, important material properties should be included in the list of arguments: the yield stress $\sigma_{Y}$ (analysis of the fracture surface shows that the local yield takes place at least at a certain part of the cycle), and a fracture toughness parameter. As the fracture toughness property it is reasonable to take Irwin's parameter $K_{I c}$ because the crack extension goes by jumps, i.e., unstably. Thus, we assume that there exists a relation of the form:

$$
\frac{d \ell}{d n}=f\left(\Delta N, R, \sigma_{Y}, K_{I c}, h\right) .
$$


The corresponding dimensions of the parameters entering (21) are as follows:

$$
[N]=\left[K_{I c}\right]=F L^{-3 / 2},\left[\sigma_{Y}\right]=F L^{-2}, \quad[h]=L,[R]=[n]=1
$$

Here again $F$ is the dimension of force, $L$ is the dimension of length. The dimension of determined quantity $d \ell / d n$ is therefore equal to $L$.

We assume as the governing parameters with independent dimensions the parameters $\Delta N$ and $\sigma_{Y}$. The dimensional analysis gives by a standard procedure

$$
\frac{d \ell}{d n}=\left(\frac{\Delta N}{\sigma_{Y}}\right)^{2} \Phi\left(\frac{\Delta N}{K_{I c}}, R, Z\right)
$$

where the dimensionless parameter

$$
Z=\frac{\sigma_{Y} \sqrt{h}}{K_{I c}}
$$

is the square root of the ratio of characteristic specimen length scale $h$ to the fracture yield length scale $\sigma_{Y}^{2} / K_{I c}^{2}$.

The evaluations show that the dimensionless parameter $\Pi_{1}=\Delta N / K_{I c}$ is small. And here we have an appropriate case to apply the technique of the analysis of asymptotic scaling laws, "advanced similarity analysis", discussed in section 2. Thus, if a finite non-zero limit of the function $\Phi$ in (23) at $\Delta N / K_{I c} \rightarrow$ 0 does exist, i.e., if there exists a complete similarity in the parameter $\Delta N / K_{I c}$, we obtain the scaling law:

$$
\frac{d \ell}{d n}=\left(\frac{\Delta N}{\sigma_{Y}}\right)^{2} \Phi_{1}(R, Z)
$$

so that the parameter $m$ in the Paris scaling law (17) is equal to 2, however the constant $A$ appears to be non-universal, depending on the specimen size. The analysis of the experimental data shows that $m=2$ is practically never the case: for some aluminum alloys $m$ is close to 2, but nevertheless always larger than 2. For the vast majority of cases $m$ is substantially larger than 2 .

Assuming incomplete similarity we obtain [32] (see also [16]):

$$
\Phi=\left(\frac{\Delta N}{K_{I c}}\right)^{\alpha(R, Z)} \Phi_{1}(R, Z),
$$

exactly the form of the experimentally observed Paris law (17) with the following expressions for the parameters of this law:

$$
A=\frac{\Phi_{1}(R, Z)}{\sigma_{Y}^{2} K_{I c}^{\alpha}}, m=2+\alpha(R, Z) .
$$

The most important conclusion of the analysis just performed is that the parameters $A$ and $m$ of the Paris law are not the material characteristics. Besides the asymmetry of the cycle $R$ they should depend on the specimen length scale. 


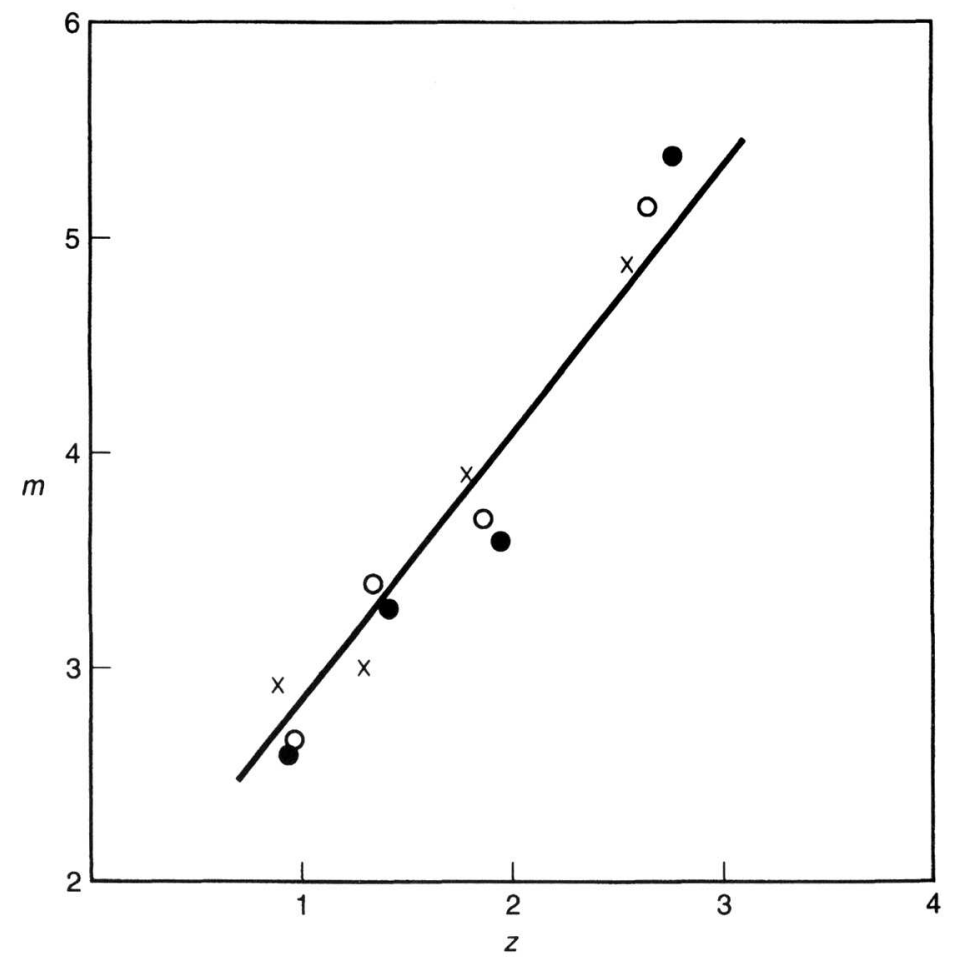

Figure 7: The dependence of the exponent in Paris law (17) on the similarity parameter $Z$ for 4340 steel (Botvina, see [32]).

This conclusion is of high importance, and it had to be checked experimentally. Indeed, it obtained a persuasive experimental confirmation. L.R. Botvina, processing the data by Heiser and Mortimer [33] (see [32]), and R.O. Ritchie [34], processing the data by Knott and Ritchie [35] (Figures 7 and 8), showed that the dependence of $m$ upon $Z$, i.e., upon the specimen size can be substantial. Therefore, using in practical structural design of the results of the standard fatigue experiments performed on small specimens can be dangerous: the real life-time of the structure can be overestimated.

I think that this example has a wider meaning. Power laws are often used in engineering practice as material properties. Characteristic examples - the calculation of $J$-integrals for plastic materials assuming the power law constitutive equation, or the evaluation of the life-time of polymeric structures assuming the power-type dependence of the fracture toughness on the crack-tip velocity. In fact, the universality of constitutive relations should be carefully checked in the the specimens of various sizes, otherwise the predictions of strength could be unreliable. 


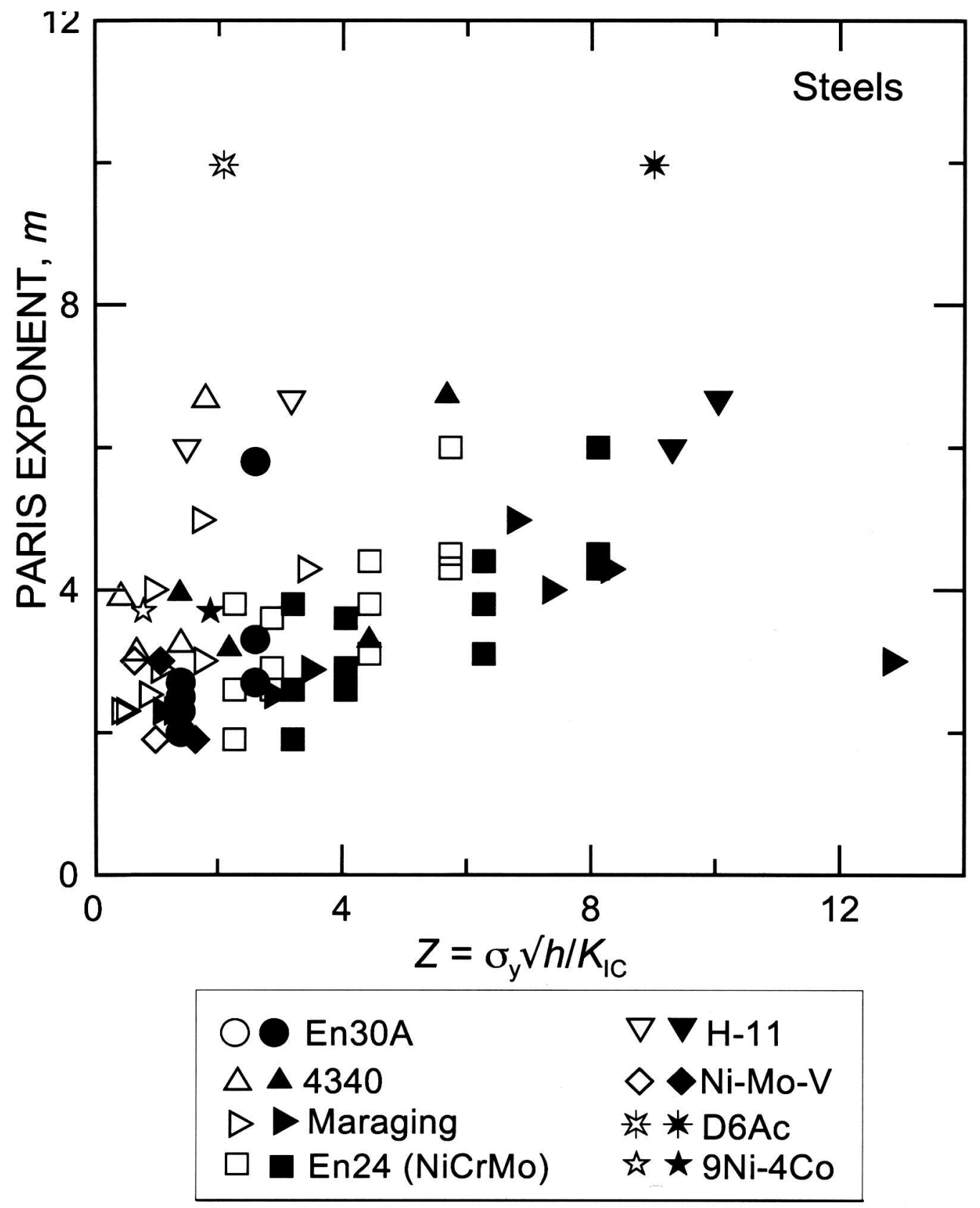

Figure 8: The dependence of the exponent in Paris law (17) on the similarity parameter $Z$ for various steels (Ritchie [35]). 


\section{Conclusion}

I want to mention in the end of my lecture a very recent result of K.B. Broberg [36], related to dynamic crack propagation and at the same time - indirectly - to incomplete similarity.

It is known that in various fields of applied mathematics over the last century, there appeared the Korteweg-de Vries equation

$$
\partial_{t} u+u \partial_{x} u+\beta \partial_{x x x}^{3} u=0,
$$

where $t$ is the time, $x$ the space coordinate, and $\beta>0$ is a constant. Equation (28) has special solitary wave solutions of the traveling wave type: $u=u(x-$ $\lambda t+c)$ :

$$
u=\frac{u_{0}}{\cos h^{2}\left(\sqrt{\frac{u_{o}}{12 \beta}} \zeta\right)}, \quad \zeta=x-\lambda t+c, \quad u_{0}=3 \lambda,
$$

so that the wave is uniformly propagating with velocity $\lambda$ and amplitude $3 \lambda$.

Transforming the variables $x=\ln \xi, t=\ln \tau$, and $c=-\ln A$ is reducing the traveling wave solution (28) to a simple self-similar form:

$$
u=\frac{12 \lambda}{2+(\eta)^{\sqrt{\lambda / \beta}}+(\eta)^{-\sqrt{\lambda / \beta}}}, \eta=\frac{\xi}{A \tau^{\lambda}} .
$$

One of the results of the remarkable work by Gardner, Greene, Kruskal and Miura [37] was that the traveling wave solutions of type (29) are intermediate asymptotics of the solutions corresponding to hump-like shapes of the initial conditions. The velocity $\lambda$ and the amplitude $u_{0}=3 \lambda$ depend on the initial conditions of the original problem for which the traveling wave is an intermediate asymptotic. The self-similar interpretation (30) of the traveling wave shows that this is exactly the case of the incomplete similarity where the power cannot be determined by dimensional analysis.

K.B. Broberg [38] found recently, using the numerical cell method which he invented, that the situation similar to traveling-wave solutions of the Kortewegde Vries equation happens also for dynamic cracks, so that for steady dynamic cracks the velocity of propagation and the amplitude are determined by initial conditions. Contrary to the case of Korteweg-de Vries solitary waves where the velocity can be an arbitrary positive number, the velocities of dynamic cracks (experimental fact!) does not exceed approximately one third of the Rayleigh velocity. What is the reason of that is yet unknown - discovery of that reason would be of fundamental interest for dynamic fracture.

I want to say in conclusion that in our time multiscale phenomena where the parameters of the same dimensions but of largely different magnitude enter the model of the phenomenon simultaneously, attract more and more attention. (The examples presented above are characteristic ones.) Nanoscience which is very popular now is one of the characteristic fields, too. Scaling and incomplete similarity will play a decisive role in studying structural strength multi-scale models, turbulence, and other branches of applied mathematics and engineering science. 


\section{Acknowledgments}

This work was supported by the Director, Office of Science, Computational and Technology Research, U.S. Department of Energy under Contract No. DEAC03-76SF00098. 


\section{References}

[1] Baržant, Z.P., Size effect in blunt fracture: concrete, rock, metal, J. of Engng. Mech. ASCE 110 (4), 518-535, 1984.

[2] Baržant, Z.P., and Planas, J. Fracture and size effect in concrete and other quasibrittle materials, CRC Press, Boston, London, New York, Washington, D.C., 1998.

[3] Baržant, Z.P., Scaling of structural strength, Hermes Penton Science, London, 2002.

[4] Baržant, Z.P., Probability distribution of energetic-statistical size effect in quasibrittle fracture, Probabilistic Engineering Mechanics 19, 307-319, 2004.

[5] Baržant, Z.P., Yavari, A., Is the cause of size effect on structural strength fractal or energetic-statistical?, Engineering Fracture Mechanics 72, 1-31, 2005.

[6] Baržant, Z.P., Scaling theory for quasibrittle structural failure, Proceedings, US Nat. Ac. Sci. 101 (37), 13400-13407, 2004.

[7] Carpinteri, A., Cusp catastrophe interpretation of fracture instability, J. of the Mechanics and Physics of Solids 37 (5), 567-582, 1989.

[8] Carpinteri, A., Scaling laws and renormalization groups for strength and toughness of disordered materials, International Journal of Solids and Structures 31 (3), 291-302, 1994.

[9] Carpinteri, A., Massabo, R., Bridged versus cohesive crack in the flexural behaviour of brittle-matrix composites, International Journal of Fracture 81, 125-145, 1996.

[10] Carpinteri, A., Strength and toughness in disordered materials: complete and incomplete similarity, in "Size-Scale Effects in the Failure Mechanisms of Materials and Structures", Carpinteri, A. (ed.), E. \& F.N. Spon, London, et. al, 3-26, 1996.

[11] Carpinteri, A., Structural Mechanics - A United Approach, E. \& F.N. Spon, London, et. al, 1997.

[12] Carpinteri, A., Chiaia, B., Cornetti, P., A scale-invariant cohesive crack model for quasi-brittle materials, Engineering Fracture Mechanics 69, 207$217,2002$.

[13] Carpinteri, A., Cornetti, P., Barpi, F., Valente, S., Cohesive crack model description of ductile to brittle size-scale transition: dimensional analysis vs. renormalization group theory, Engineering Fracture Mechanics 70, 1809-1839, 2003. 
[14] Barenblatt, G.I., Zeldovich, Ya.B., Intermediate asymptotics in mathematical physics, Russian Mathematical Surveys 26 (2), 45-61, 1971.

[15] Barenblatt, G.I., Zeldovich, Ya.B., Self-similar solutions as intermediate asymptotics, Ann. Rev. Fluid Mech. 4, 285-312, 1972.

[16] Barenblatt, G.I., Scaling, self-similarity, and intermediate asymptotics, Cambridge University Press, 1996.

[17] Barenblatt, G.I., Scaling, Cambridge University Press, 2003.

[18] Taylor, G.I., The formation of a blast wave by a very intense explosion, Report RC-210, Civil Defense Research Committee, 27 June 1941.

[19] Taylor, G.I., The formation of a blast wave by a very intense explosion. II. The atomic explosion of 1945, Proc. Roy. Soc. A201, 175-186, 1950.

[20] Barenblatt, G.I., On the equilibrium cracks formed in brittle fracture, J. Appl. Math. Mech. (PMM) (3) 434-444, (4) 706-721, (5) 893-900, 1959.

[21] Irwin, G.R., Fracture made transition for a crack traversing a plate, Trans. ASME, Ser. D 82, 417-425, 1960.

[22] Panasyuk, V.V., Limiting equilibrium in brittle bodies with cracks, Naukova Dumka, Kiev, 1968,

[23] Barenblatt, G.I., On certain problems of the theory of elasticity, which arise in the theory of the hydraulic fracture of the oil stratum, J. Appl. Math. Mech. (PMM) 20 (4), 475-486, 1956.

[24] Roesler, F., Brittle fracture near equilibrium, Proc. Phys. Soc. B69, 981992, 1956.

[25] Benbow, J.J., Cone cracks in fused silica, Proc. Phys. Soc. B75, 697-699, 1960.

[26] Goldenfeld, N.D., Lectures on Phase Transition and the Renormalization Group, Perseus Publishing, Reading, 1992.

[27] Mandelbrot, B., Fractals, Form, Chance and Dimension, W.H. Freeman and Co., San Francisco, 1977.

[28] Mandelbrot, B., Les objects fractals: forme, hasard et dimension, Flammarion, Paris, 1975.

[29] Mandelbrot, B., The Fractal Geometry of Nature, W.H. Freeman and Co., San Francisco, 1982.

[30] Paris, P.C., Erdogan, F., A critical analysis of crack propagation laws, J. Basic Engineering Trans. ASME, Ser. D. 85, 528-534, 1963. 
[31] Paris, P.C., Gomez, M.P., Anderson, W.P., A traditional analytic theory of fatigue. The Trend in Engineering 13, 9-14, 1961.

[32] Barenblatt, G.I., Botvina, L.R., Incomplete self-similarity of fatigue in the linear range of crack growth, Fatigue of Engineering Materials and Structures 3, 193-212, 1981.

[33] Heiser, F.A., Mortimer, W., Effects of thickness and orientation on fatigue crack growth rate in 4340 steel, Met. Trans. 3, 2119-2123, 1972.

[34] Ritchie, R.O., Self-similarity and fatigue-crack growth, International Journal of Fracture, 2004 (submitted).

[35] Ritchie, R.O., Knott, J.F., Mechanisms of fatigue crack growth in low alloy steel, Acta Metallurgica 21, 639-648, 1973.

[36] Gardner, C.S.J., Greene, J.M., Kruskal, M.D., Miura, R.M., A method for solving the Korteweg-de Vries equation, Phys. Rev. Lett. 19, 1095-1097, 1967.

[37] Broberg, K.B., Significance of morphological changes at a propagating crack edge, International Journal of Fracture, 2004 (in press).

[38] Botvina, L.R., The Kinetics of Fracture of Structural Materials, Nauka, Moscow, 1989. 